\title{
Mhd Accretion Disk Winds And The Blazar Sequence
}

\section{Stella S. Boula*}

Department of Physics, National and Kapodistrian University of Athens, Panepistimiopolis, GR 15783 Zografos, Greece

E-mail: stboula@phys.uoa.gr

\section{Demosthenes Kazanas}

NASA Goddard Space Flight Center, Greenbelt, MD, United States

\section{Apostolos Mastichiadis}

Department of Physics, National and Kapodistrian University of Athens, Panepistimiopolis, GR 15783 Zografos, Greece

Fermi telescope has detected a significant number of AGNs to allow statistical treatment of their properties. Among others, it confirmed the 'Blazar Divide' in FSRQs and BL Lacs according to their gamma-ray spectral index and luminosity. We investigate this classification by proposing a model which reproduces in detail the broadband Blazar spectra and their statistical properties based on the physical parameters of their MHD accretion disk winds. This model describes the distribution of matter and magnetic fields in AGN at least over 5 decades in radius and it provides the vestiges of an account of the observed Blazar classification in terms of a single parameter, their mass accretion rate.

High Energy Phenomena in Relativistic Outflows VII - HEPRO VII

9-12 July 2019

Facultat de Fisica, Universitat de Barcelona, Spain

\footnotetext{
* Speaker.

${ }^{\dagger}$ State Scholarships Foundation (IKY) fellow
} 


\section{Accretion Disk MHD Winds: A Unifying AGN Structure.}

Blazars (Flat Spectrum Radio Quasars and BL Lacs) are types of Active Galactic Nuclei (AGN) with relativistic jets pointing toward us. The relativistic bulk motion of these jets amplifies their radiation, which in general dominates their disk emission, making these objects extremely bright and therefore visible at large redshifts. The Blazar jet spectra are non-thermal, implying emission by relativistic particle distributions. They consist of two broad "humps", one that spans from radio to optical-UV (and occasionally X-ray) bands and another one that extends from X-rays to multi-GeV and, on occasion, to $\mathrm{TeV} \gamma$-rays. The former is thought to be due to synchrotron emission by non-thermal electrons, while the latter by inverse Compton (IC) scattering of the relativistic electrons on synchrotron or external photons. The size of the emitting region is not well constrained; the typical size estimates obtained from temporal variability arguments are affected by relativistic plasma motion and radiative cooling. Certain patterns in the spectral features became apparent and are now known as the "Blazar Sequence" [1, 2, 3, 4]. Blazars become redder with increasing bolometric luminosity $L_{\mathrm{bol}}$, in that their synchrotron peak frequency $v_{\mathrm{pk}}^{\mathrm{syn}}$ decreases as $L_{\mathrm{bol}}$ increases; at the same time their Compton dominance (CD; i.e. the ratio of their IC to synchrotron luminosities) increases and so do their $\gamma$-ray spectral indices. The Blazar Sequence, established originally with 132 objects out of which only 33 were detected in high energy $\gamma$-rays, was supplanted with the launch of Fermi and the discovery of more than $1000 \gamma$-ray Blazars in the 2nd, 3rd and 4th Fermi Blazar Catalogs [5, 6, 7]. These compilations provided novel correlations that replaced those of the original Blazar Sequence. Most importantly, these correlations were found to be independent of the source luminosity, implying that the underlying physics are related to dimensionless parameters.

The apparently optically thin Blazar GeV emission suggests its location to be far from the accreting black hole $(\mathrm{BH})$, out to a distance as large as $10^{6} R_{S} \sim 10 \mathrm{pc}$ [8] (where $R_{S}$ is the Schwarzschild radius ). One might argue that at this distance the high energy emission is not connected to the AGN properties. However, the AGN torii (dusty, molecular structures of height/radius ratios $z / D \simeq 1$ ) invoked in the unification of the radio quiet or radio loud AGN subclasses [9], are of similar scales and, as we argue, play a significant role in Blazar physics. To reconcile the discrepancy of the torii geometry expected by hydrostatic equilibrium with their thermal and inferred Keplerian velocities, [10] proposed that these torii are, in fact, MHD accretion disk winds [11, 12]; these are launched across the entire disk, from the $\mathrm{BH}$ vicinity of a few $R_{S}$ to the $\mathrm{BH}$ influence radius $D \sim(c / \sigma)^{2} R_{S} \sim 10^{6} R_{S} \sim 10 p c$ (for $M_{B H} \simeq 10^{8} M_{\odot}$ ). Furthermore, the discovery of blueshifted absorption features in the AGN X-ray spectra, the so-called Warm Absorbers (WA) and their successful modeling as photoionized MHD winds that extend to $r \sim 10^{6} R_{S}$ [13, 14], established the combined AGN WA-torii as a single entity, namely MHD winds launched across the entire accretion disk domain $\left[R_{S} \sim D\right]$. Finally, modeling the absorbers of the Galactic BH GRO $1655-40$ with the same winds [15] suggests the possibility of their presence in any accreting BH.

In our work [11] we reproduced a theoretical Blazar Sequence by varying only one parameter, "namely the mass accretion rate. Here we will show the dependence of our results on the other free parameters of this problem (e.g. the efficiencies for the magnetic field strength, the electrons power, the efficiency for the external photon field). 


\section{Model}

The broader morphology of the non-thermal Blazar SED depends on the ratio of the magnetic to photon energy densities. Here we assume that the external photon field is related to photons which are scattered on accretion disk wind particles. In order to calculate the Blazar SED we solve the kinetic equations of electrons and photons as described by [16]. We assume that electrons are injected in the source with a broken power law distribution given by

$$
\mathrm{Q}_{\mathrm{e}}= \begin{cases}k_{\mathrm{e}_{1}} \gamma^{-p} & \text { for } \gamma_{\min } \leq \gamma \leq \gamma_{\mathrm{cool}}, \\ k_{\mathrm{e}_{2}} \gamma^{-q} e^{-\gamma / \gamma_{\max }} & \text { for } \gamma_{\mathrm{cool}} \leq \gamma \leq \gamma_{\max },\end{cases}
$$

where $L_{\mathrm{e}}^{\mathrm{inj}}=m_{\mathrm{e}} c^{2} \int_{\gamma_{\min }}^{\gamma_{\max }} Q_{\mathrm{e}}(\gamma) \gamma \mathrm{d} \gamma=\eta_{e} P_{\text {acc }}$, with $\eta_{e}$ is a proportionality constant $\left(\eta_{e}<1\right)$. Here $P_{\text {acc }}=\dot{m} \mathscr{M} L_{\mathrm{Edd}}$, with $\dot{m}$ the mass accretion rate normalized to the Eddington one and $\mathscr{M}=$ $M_{\mathrm{BH}} / M_{\odot}$ where $M_{\mathrm{BH}}$ is the mass of the black hole. The electron distribution is characterized by $\gamma_{\min }$ and $\gamma_{\max }$ (the minimum and maximum electrons Lorentz factors), $p$ and $q$ the indices before and after the break respectively, $\gamma_{\mathrm{cool}}=\frac{3 m_{\mathrm{e}} c^{2}}{4 \sigma_{\tau} c t_{\mathrm{dyn}} U_{\mathrm{tot}}}$, where $t_{\mathrm{dyn}}=\frac{R_{\mathrm{b}}}{c}$ is the dynamic time scale, while $U_{\text {tot }}$ is the total energy density and it is given by $U_{\text {tot }}=U_{\text {ext }}+U_{\mathrm{B}}+U_{\mathrm{SSC}}$, where $U_{\text {ext }}=\Gamma^{2} U_{\text {sc }}$ is the energy density of the scattered photons on the wind particles as measured in the co-moving frame ( $\Gamma$ is the bulk Lorentz factor of the source), $U_{\mathrm{B}}$ is the energy density of the magnetic field at position $\mathrm{z}$ and $U_{\mathrm{SSC}}$ is the energy density of the produced synchrotron photons. In order to calculate the SED of scattered photons we have assumed that the disk emits like a black body characterized by a temperature $T_{\text {disk }}$. As we pointed at [11], all input parameters required for the calculation of the spectrum are scaled with $\dot{m}$ and $\mathscr{M}$. Thus one can write:

$$
\begin{gathered}
\mathrm{U}_{\mathrm{B}} \propto \eta_{\mathrm{b}} \dot{m} \mathscr{M}^{-1}, \\
\mathrm{U}_{\mathrm{ext}} \propto \mathrm{U}_{\mathrm{sc}} \propto \varepsilon \dot{m}^{\alpha+1} \mathscr{M}^{-1}(\alpha=1 \text { for } \dot{m} \geq 0.1 \text { and } \alpha=2 \text { for } \dot{m}<0.1), \\
\gamma_{\mathrm{cool}} \propto \dot{m}^{-1}\left(1+\dot{m}^{\alpha}\right)^{-1}, \\
\mathrm{~L}_{\mathrm{e} j} \propto \eta_{\mathrm{e}} \dot{m} \mathscr{M},
\end{gathered}
$$

where $\eta_{\mathrm{b}}$ is is a proportionality constant and $\varepsilon$ is the efficiency of the conversion of the accreting mass into radiation.

\section{Results}

In our work we relate the mass accretion rate to the Blazar type. We assume that the case of $\dot{m}>0.1$ corresponds to a radiatively efficient disk and therefore to a FSRQ object, where $U_{\text {ext }}$ is high and the source is Compton dominated. On the other hand, the case of $\dot{m} \ll 0.1$ corresponds to a disk in an ADAF state with low $U_{\text {ext }}$ and as a result SSC starts dominating over EC scattering and thus it represents a BL Lac object [17]. Furthermore, the observed peak of the synchrotron component for electrons with Lorentz factor $\gamma_{\text {cool }}$ will appear at $v_{\mathrm{syn}} \propto \delta B \gamma_{\text {cool }}^{2}$. Using the relations described in the previous section, we find

$$
v_{\text {syn }} \propto \mathscr{M}^{-1 / 2} \dot{m}^{-3 / 2}\left(1+\dot{m}^{\alpha}\right)^{-2} .
$$

As $\dot{m}$ increases, the decrease of $\gamma_{\text {cool }}$ shifts $v_{\text {syn }}$ to lower frequencies, despite the magnetic field strength increase. On the other hand, a low value of $\dot{m}$ corresponds to lower values of the magnetic 
field strength and the corresponding increase in $\gamma_{\text {cool }}$ leads to a higher value for $v_{\text {syn }}$.

In Fig. 1 we present the results of our model calculations in comparison with the observational data of $\alpha_{\gamma} v s . v_{\text {syn }}$ of 3LAC (fixed observation band between $v \sim 10^{22}-10^{24} \mathrm{~Hz}$ ). We have chosen a value for the black hole mass $\mathscr{M}=10^{9}$ and three values of $\log \dot{m}$, namely $-0.5,-1.5$ and -2.5 . The location of the emitting source was assumed to be at $z=1 \mathrm{pc}$. Assuming that soft photons - which are scattered on the accretion disk wind particles - are isotropically distributed between radii $R_{1}=0.03 \mathrm{pc}$ and $R_{2}=10 \mathrm{pc}$, we calculate the external photon field energy density which depends on the wind's optical depth. For the efficiencies we adopt the values $\eta_{\mathrm{e}}=0.1, \eta_{\mathrm{b}}=0.05$ and $\varepsilon=0.5$ respectively. The indices for the electron distribution are $p=2$ and $q=2.75$. The maximum Lorentz factor we assume is $\gamma_{\max }=10^{4} \gamma_{\text {cool }}$, while the bulk Lorentz factor is $\Gamma=30$ and the Doppler factor is $\delta=15$. The characteristic temperature of the disk is $T_{\text {disk }}=3 \times 10^{3} \mathrm{~K}$.

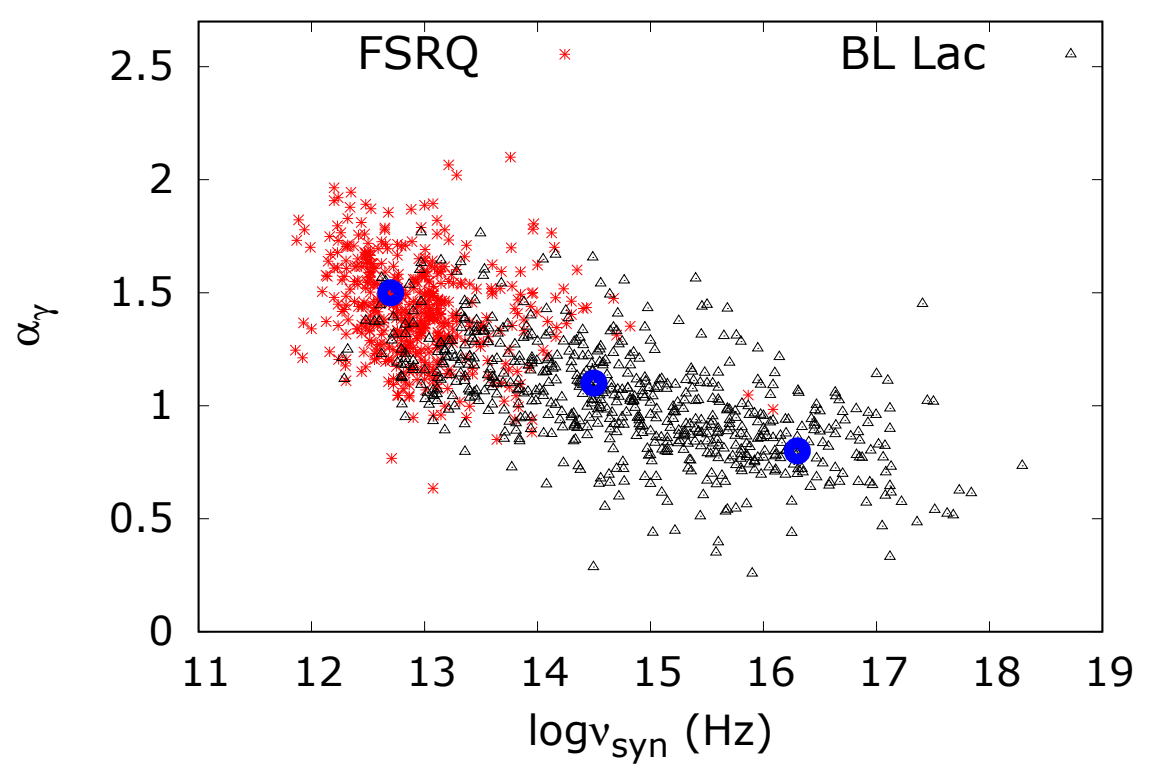

Figure 1: The variation of the $\gamma$-ray photon index, $\alpha_{\gamma}$, as a function of the frequency of the synchrotron peak, $v_{\mathrm{syn}}^{\mathrm{pk}}$, as shown in the 3LAC compilation [6]. The circular points show our model results for $\log \dot{m}$ values of $-0.5,-1.5$ and -2.5 (left to right).

In Figures 2, 3, 5 and 6 we show the dependence on the efficiencies $\eta_{b}, \eta_{e}$ and $\varepsilon$ of the results that are depicted in Figure 1. We vary these parameters and we calculate the SEDs for various combinations. We let them take the values $\eta_{b}=0.025,0.05,0.1, \eta_{e}=0.05,0.1,0.2$ and $\varepsilon=0.25,0,5,0.75$ for three different values of $\dot{m}$ (the same as Figure 1). In Figure 4 we show how the $\gamma$-ray photon index $\alpha_{\gamma}$ depends on the maximum electrons Lorentz factor $\gamma_{\max }$ and on the slope $q$. These two parameters are related to the details of the particles acceleration. The slope $p$ before the cooling break does not affect the value of $\alpha_{\gamma}$. As it can be seen, the calculated spread of the plane $\alpha_{\gamma}$ versus $v_{s y n}$ is similar to the observational one. Furthermore, one can see that there is a trend between the different quantities (i.e. $L_{s y n}$ vs $L_{I C S}, v_{s y n}$ vs $v_{I C S}$ or the Compton Dominance and the peak frequencies). 

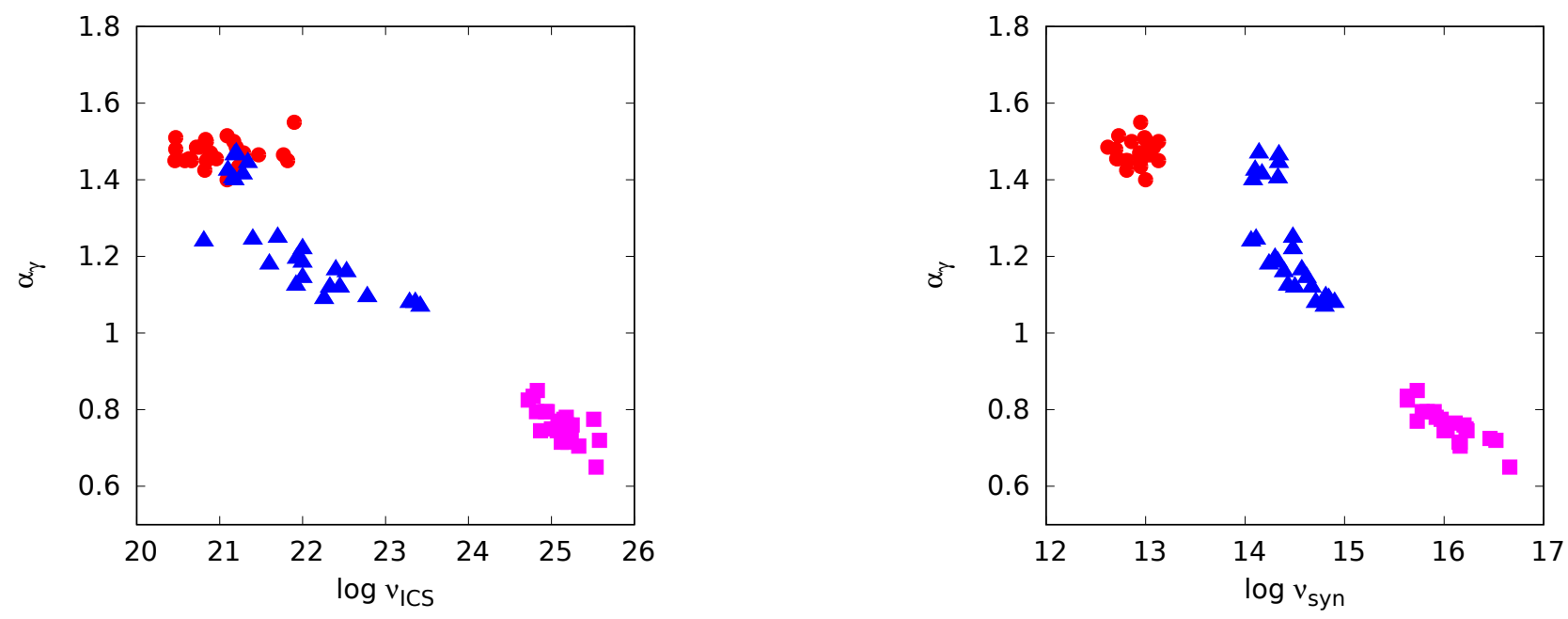

Figure 2: Left: Peak of inverse Compton scattering frequency versus the $\gamma$-ray photon index $\alpha_{\gamma}$. Right: Peak of synchrotron frequency versus the $\gamma$-ray photon index $\alpha_{\gamma}$. The red circular points represent FSRQs $(\dot{m}=-0.5)$, the blue triangular points represent low synchrotron peak BL Lac objects $(\dot{m}=-1.5)$ and the magenta square points represent intermediate and high synchrotron peak BL Lac objects $(\dot{m}=-2.5)$.

\section{Discussion}

In this work we follow our model [11] where we have shown that a one-zone leptonic model with a simple scaling of its input parameters on the (dimensionless) mass accretion rate can reproduce the basic trends of the Blazar Sequence and extend it to examine the effects of the secondary parameters such as the various efficiencies, the maximum energy of the electrons and the slope above the cooling break. In Fig. 1 we present the observational data of the 3LAC [6] in comparison with the results of our theoretical Blazar Sequence. While the variation of our primary parameter the mass accretion rate $\dot{m}$ gives the correct trend to the data, small variations of the other parameters of the problem reproduce the observational data spread. In Fig. 2 we compare the $\gamma$-ray photon index to the synchrotron peak frequency (as in the Fig. 17 from [6]) and to the inverse Compton peak frequency (as in Fig. 3 from [3]). We can see that there is a characteristic trend which is achieved by varying $\dot{m}$ and is independent from the rest of the free parameters. Figure 3 is in agreement with the observational data of Fig. 9 from [7]. The 4th catalog has more sources and as we can see FSRQs present higher values for $\gamma$-ray photon indices. In order to reproduce this trend we vary the parameters of the electron injection $\left(\gamma_{\max }, q\right)$, and find that while the peak of the synchrotron frequency does not change, the slope of the SED is affected (Fig. 4). In Fig. 5 we found that the plane between the synchrotron and inverse Compton scattering luminosities shows a linear trend. In the same figure, in the right panel where the peak frequencies are depicted, one can see that FSRQs are affected from the synchrotron self absorption which causes the values of $v_{\text {syn }}$ to be around $10^{13} \mathrm{~Hz}$, while the low and intermediate peaked synchrotron BL Lac objects 


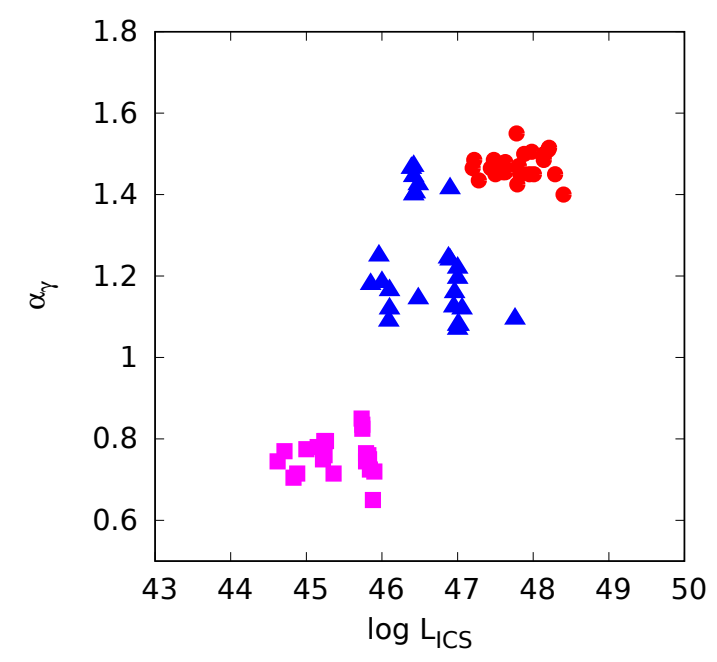

Figure 3: Photon index versus $\gamma$-ray luminosity for the different blazar classes, the points are the same as Figure 2

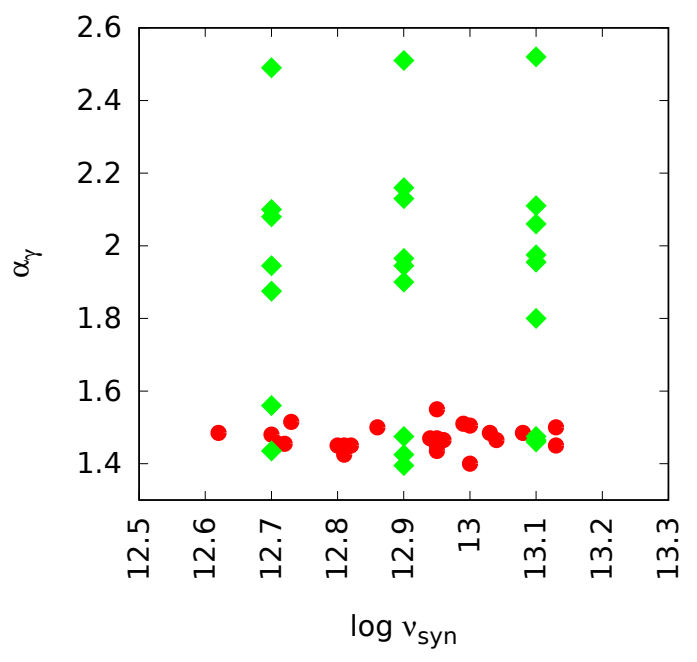

Figure 4: Peak of synchrotron frequency versus the $\gamma$-ray photon index $\alpha_{\gamma}$ in the case of FSRQs $(\dot{m}=-0.5)$. The red circles show the same points as Figure 2, the green rhombus points show the vertical dependence of a point when we vary electron index $q(q=2,2.75,3)$ and electrons maximum Lorentz factor $\gamma_{\max }\left(\gamma_{\text {cool }} \leq \gamma_{\max } \leq 10^{4} \gamma_{\text {cool }}\right)$. 

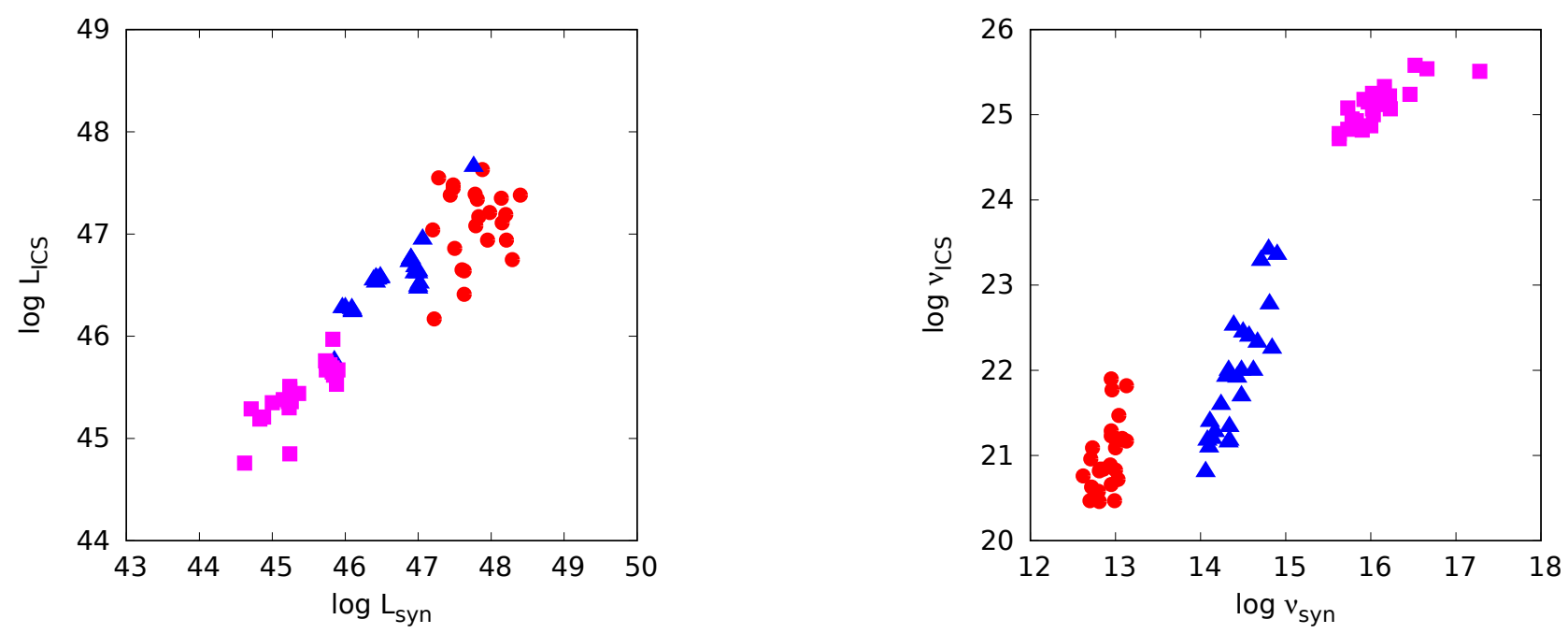

Figure 5: Left: Peak synchrotron luminosity versus peak inverse Compton scattering luminosity. Right: Peak synchrotron frequency versus peak inverse Compton scattering frequency: Symbols are the same as in Figure 2.
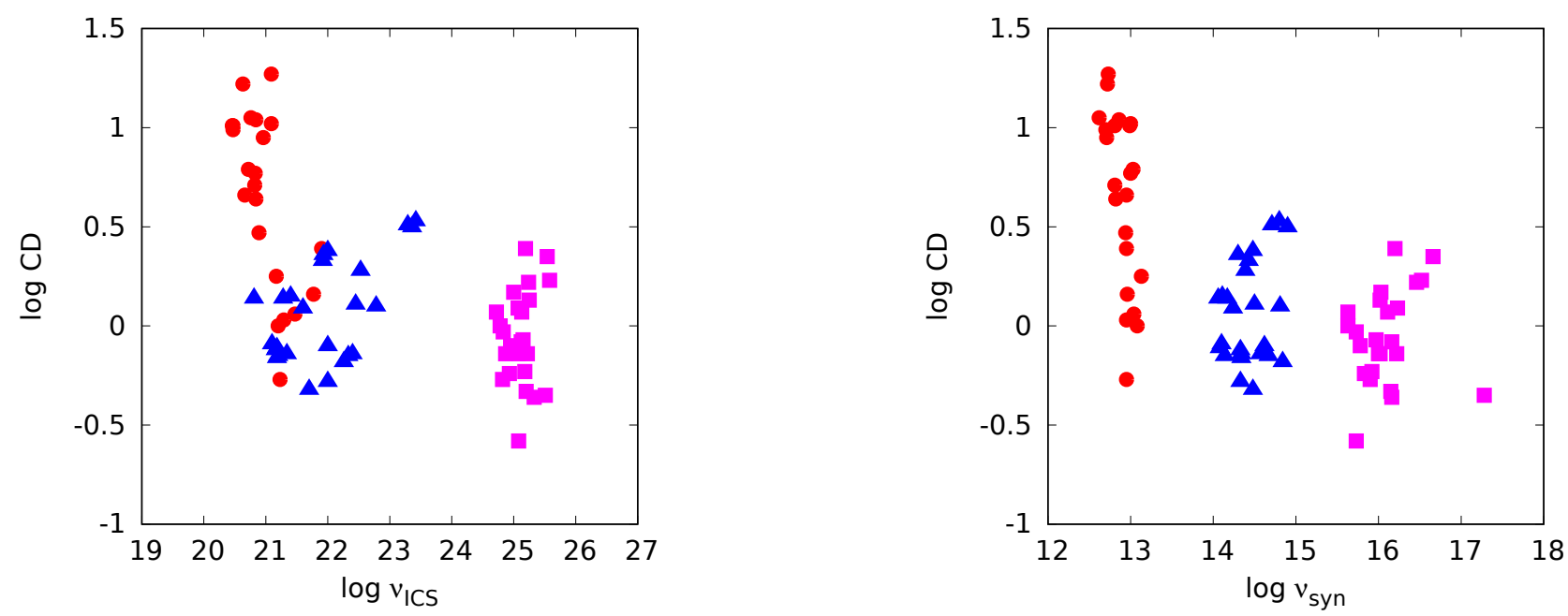

Figure 6: Left: Compton dominance (i.e. $L_{\mathrm{ICS}} / L_{\mathrm{syn}}$ ) versus peak inverse Compton scattering frequency. Right: Compton dominance versus peak synchrotron frequency: Symbols are the same as in Figure 2.

show a spread; high peaked synchrotron BL Lac objects show a $v_{\text {ICS }}$ around $10^{25} \mathrm{~Hz}$ because of the Klein - Nishina cutoff. The left plot from Fig. 6 is the analogous as Fig. 5 from [3]. For completeness reasons the right panel shows the Compton Dominance (CD) versus $v_{\text {ICS. FSRQs }}$ 
are Compton dominated and the external photon field plays a key role on its value. For example, when the magnetic field is large and the external photon field small, then CD is less than one. In the case of BL Lac objects, the energy density of the external photon field is low, SSC dominates and in this case there is less variation in the $\mathrm{CD}$. Therefore it is interesting this simply preliminary analysis can reproduce quite well the trends of the observational data.

\section{Acknowledgments}

SB: This research is co-financed by Greece and the European Union (European Social Fund-ESF) through the Operational Programme "Human Resources Development, Education and Lifelong Learning" in the context of the project "Strengthening Human Resources Research Potential via Doctorate Research" (MIS-5000432), implemented by the State Scholarships Foundation (IKY).

\section{References}

[1] G. Fossati, L. Maraschi, A. Celotti, A. Comastri and G. Ghisellini, A unifying view of the spectral energy distributions of blazars, 299 (1998) 433 [astro-ph/9804103].

[2] G. Ghisellini, A. Celotti, G. Fossati, L. Maraschi and A. Comastri, A theoretical unifying scheme for gamma-ray bright blazars, 301 (1998) 451 [astro-ph/9807317].

[3] J. D. Finke, Compton Dominance and the Blazar Sequence, 763 (2013) 134 [1212 . 0869 ].

[4] G. Ghisellini, C. Righi, L. Costamante and F. Tavecchio, The Fermi blazar sequence, 469 (2017) 255 [1702.02571].

[5] M. Ackermann, M. Ajello, A. Allafort, E. Antolini, W. B. Atwood, M. Axelsson et al., The Second Catalog of Active Galactic Nuclei Detected by the Fermi Large Area Telescope, 743 (2011) 171 [1108.1420].

[6] M. Ackermann, M. Ajello, W. B. Atwood, L. Baldini, J. Ballet, G. Barbiellini et al., The Third Catalog of Active Galactic Nuclei Detected by the Fermi Large Area Telescope, 810 (2015) 14 [1501.06054].

[7] The Fermi-LAT collaboration, The Fourth Catalog of Active Galactic Nuclei Detected by the Fermi Large Area Telescope, arXiv e-prints (2019) arXiv:1905.10771 [1905.10771].

[8] A. P. Marscher, S. G. Jorstad, V. M. Larionov, M. F. Aller, H. D. Aller, A. Lähteenmäki et al., Probing the Inner Jet of the Quasar PKS 1510-089 with Multi-Waveband Monitoring During Strong Gamma-Ray Activity, 710 (2010) L126 [1001.2574].

[9] R. R. J. Antonucci and J. S. Miller, Spectropolarimetry and the nature of NGC 1068, 297 (1985) 621.

[10] A. Konigl and J. F. Kartje, Disk-driven hydromagnetic winds as a key ingredient of active galactic nuclei unification schemes, 434 (1994) 446.

[11] S. Boula, D. Kazanas and A. Mastichiadis, Accretion disc MHD winds and blazar classification, 482 (2019) L80 [1810.01796].

[12] J. Contopoulos and R. V. E. Lovelace, Magnetically Driven Jets and Winds: Exact Solutions, 429 (1994) 139.

[13] E. Behar, Density Profiles in Seyfert Outflows, 703 (2009) 1346 [0 908 . 0539 ]. 
[14] K. Fukumura, D. Kazanas, I. Contopoulos and E. Behar, Magnetohydrodynamic Accretion Disk Winds as X-ray Absorbers in Active Galactic Nuclei, 715 (2010) 636 [0910 . 3001 ].

[15] K. Fukumura, D. Kazanas, C. Shrader, E. Behar, F. Tombesi and I. Contopoulos, Magnetic origin of black hole winds across the mass scale, Nature Astronomy 1 (2017) 0062 [1702 . 02197].

[16] A. Mastichiadis and J. G. Kirk, Self-consistent particle acceleration in active galactic nuclei., 295 (1995) 613.

[17] G. Ghisellini, L. Maraschi and F. Tavecchio, The Fermi blazars' divide, 396 (2009) L105 [0903.2043]. 